\title{
A DECISION SUPPORT SYSTEM FOR SDH NETWORK PLANNING CONSIDERING EQUIPMENT MODULARITY ASPECTS
}

\author{
Anilton Salles Garcia \\ Informatic Department, Federal University of Espirito Santo - UFES \\ P. O. Box 19044, ZIP 29060-970, Vitoria, ES, Brazil \\ Gustavo Balestrero Zanandrea \\ Tubarao Port Superintendence, Vale do Rio Doce Company - CVRD \\ O. Box 1078, ZIP 29072-000, Vitoria, ES, Brazil
}

\begin{abstract}
RESUMO: O processo de digitalização das redes telefônicas está permitindo uma rápida introdução dos novos serviços de telecomunicações na rede. Esses serviços estão exigindo uma rede de transporte confiável, assim como, sistemas de transmissão de alta capacidade com elevados niveis de qualidade. Visando atender todos esses requisitos, o ITU-T padronizou a SDH (Synchronous Digital Hierarchy - Hierarquia Digital Síncrona) para a rede de transporte. Esse trabalho apresenta um modelo de otimização combinatória para o Problema Estático do Enfeixamento, que determina a disposição lógica dos enlaces ópticos e dos equipamentos de transmissão, considerando os aspectos de modularidade. Esse problema é modelado como um problema de fluxo em rede de multi-produtos e o modelo resultante tem uma característica de programação linear inteira mista, e sua resolução combina métodos exatos com heurísticas especializadas.
\end{abstract}

\begin{abstract}
The digitization process of the telephone networks is allowing a quick introduction of the new sophisticated telecommunications services into the network. These services are demanding a reliable transport network, as well as, high capacity transmission systems with high quality levels. Wishing to attempt all these requirements, the ITU-T has standardized the SDH (Synchronous Digital Hierarchy) for the transport network. This paper proposes a combinatorial optimization model for the Static Bundling Problem, that consists of the logical design of the optical links and the transmission equipment, considering the modularity aspects. This problem is modeled as a multicommodity network flow problem and the resulting model has a mixed integer linear programming feature that is solved combining exact methods with specialized heuristics.
\end{abstract}

Key words: SDH, Telecommunication Network Planning, Optimization, Modularity.

The digitization process of the telephone networks is allowing a quick introduction of the new sophisticated telecommunications services into the network. These services are demanding a reliable transport network, as well as, high capacity transmission systems with high quality levels. Wishing to attempt all these requirements, the ITU-T has standardized the SDH (Synchronous Digital Hierarchy) for the transport network. This paper proposes a combinatorial optimization model for the Static Bundling Problem, that consists of the logical design of the optical links and the transmission equipment, considering the modularity aspects. This problem is modeled as a multicommodity network flow problem and the resulting model has a mixed integer linear programming feature that is solved combining exact methods with specialized heuristics.

\section{INTRODUCTION}

Since the decade of 1970 , the development of the telecommunication networks are being impacted by the optical transmission. Due to the use of this technology, the costs of the transmission systems are being reduced. When compared to copper cable and radio systems, the optical fiber has many advantages such as higher capacity, higher reliability, longer repeater spacing, greater security, smaller size, less weight, unlimited growth potential. These properties actually make the optical fiber the best choice for the transmission media.

The modern countries are moving towards the planning and the deployment of a new transport network that can offer a reliable and flexible signal transport capability needed to support the new telecommunications services. These new services, basically formed by high speed data transfer, require the use of high capacity optical systems. These systems concentrate the traffic, that is very interesting in an economical viewpoint, due to the economy of scale, but can bring severe damage to the quality of the services in case of a single failure. Owing to this, the network reliability is a very important planning aspect. A single failure of equipment or media can let thousands of persons without communication. A new concept, complementary to the reliability, was created to deal with these new problems: Survivability.

Survivability is defined as the percentage of total demands still intact in case of a network failure. This failure can be 
at an optical link or at a hub. Two survivability measures that are often used are the average survivability and the worst case survivability. The worst case survivability is the lowest percentage of circuits surviving over all possible, when occurring single failures. It allows engineers and planners to locate the most vulnerable segment of the network and it is typically used for strategic planning. The average survivability measurement is often used when the failure probability of each network component and the average time to restore service due to this failure are known [1].

To support all these new network requirements, it is necessary to develop a decision support system for the SDH (Synchronous Digital Hierarchy) network planning.

The most important problem in the transport network planning, the Bundling, can be solved in a more efficient way using the SDH technology. In this paper, we present a combinatorial optimization model for the SDH Static Bundling Problem, that considers the equipment modularity aspects. As an example, a case study, based on a medium size Brazilian Metropolitan Area Network will also be presented.

\section{THE SYNCHRONOUS DIGITAL HIERARCHY}

The SDH is specified by ITU-T (International Telecommunications Union - Transmission Section) in recommendations G.707, G.708 and G.709, based on the SONET (Synchronous Optical NETwork) format, an important job of Bellcore (Bell Communications Research) for establishing a new digital transmission technology. It has many advantages over the PDH (Plesiochronous Digital Hierarchy), nowadays used, such as [1]:

1. It is a standard format for the whole world;
2. It allows direct access to the tributaries, without using intermediary rates;

3. It makes easier to increase the transmission rates, because it is based on a byte interleaving process;

4. It is compatible with the ATM (Asynchronous Transfer Mode);

5. It brings compatibility between vendors;

6. It permits a centralized management and operation control.

In the SDH multiplexing process, shown in Figure 1, the tributaries, that can be a variety of PDH signals, are mapped on the Payload of a Low-Order Virtual Container (LVC), that can be VC-11, VC-12, VC-2, VC-3. Some management information are associated to this $\mathrm{LVC}$ in the Path Overhead (POH). Joining several LVCs, a HighOrder Virtual Container (HVC), VC-3 and VC-4, is formed. Adding the Section Overhead (SOH), the FirstOrder Synchronous Transfer Mode (STM-1) is formed. The higher order STMs are obtained by the synchronous multiplexing, i.e., the byte interleaving

The SDH equipment are classified in three types [1]:

1. TM (Terminal Multiplexer): it multiplexes a variety of low-speed signals (tributaries) to a high-speed one, making the electrical-optical conversion, and then places the resultant optical signal onto the fiber;

2. ADM (Add-Drop Multiplexer): it provides not only the network multiplexing function (as does the TM), but also an automatic add-drop capability and remote settings;

3. SDXC (Synchronous Digital Cross-Connector): it terminates optical signals and automatically crossconnect constituent signals (tributaries) according to a

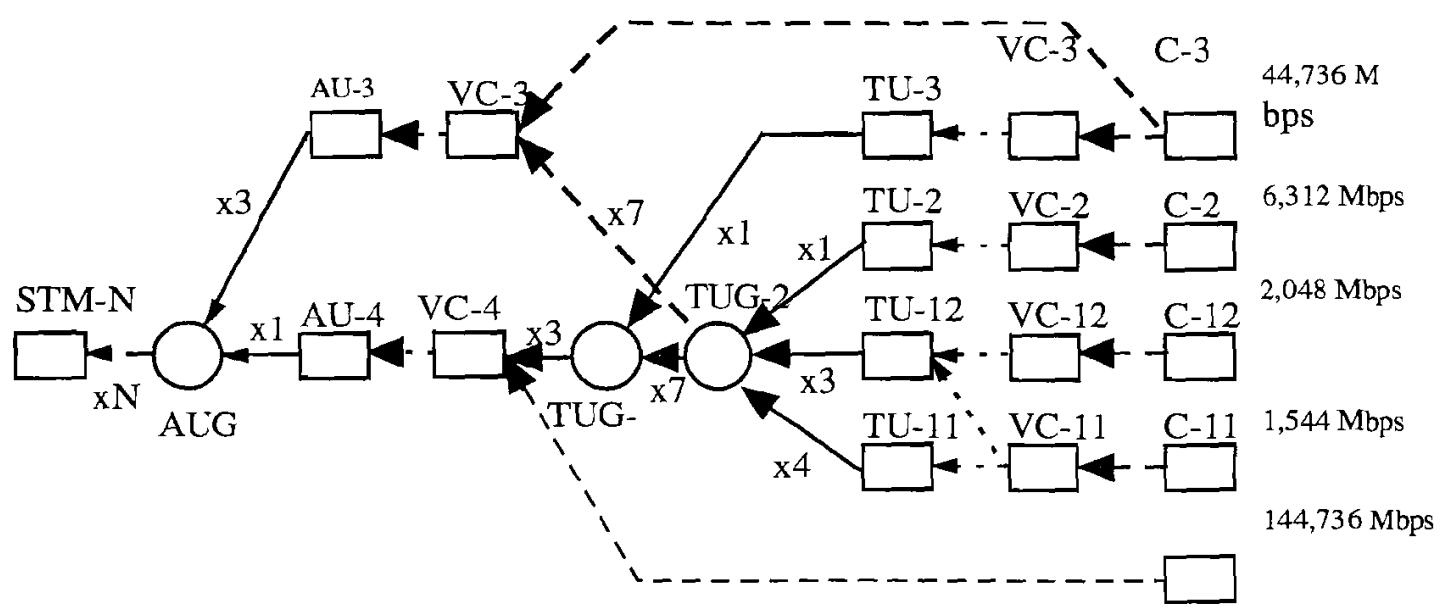

Figure 1 - Multiplexing Process 
stored map. The SDXC integrates multiple functionalities such as signal add-drop, signal crossconnect and multiplexing / demultiplexing into a single network element.

The NEs (Network Elements) can be logically displaced in several ways, forming different elementary topologies for the SDH Transport Network. There are three elementary categories of topologies [1], [3]:

1. Point-to-point: the subnetwork consists of a pair of nodes that are connected by two physically diverse transmission paths. In case of a failure of the working path, the affected traffic of the single point-to-point is switched to the protection path;

2. Ring: the subnetwork consists of a collection of nodes forming a closed loop. In case of a failure, the demands of any affected traffic pair within the ring subnetwork can be rerouted in the opposite direction. A special type is the Self-Healing Ring (SHR), that provides redundant bandwidth and/or network equipment so disrupted services can be automatically restored following networks failures. It can be an Unidirectional SHR or a Bi-directional SHR. Wishing to improve the survivability level, it can be implemented using four optical fibers.

3. Mesh: a mesh subnetwork is one where the degree of connectivity (the number of links coincident on that node) of all the nodes exceeds two. In case of a failure, the affected traffic can be rerouted over a variety of diverse paths.

The use of a protection architecture, defined as an autonomously subnetwork that carries digital signals, as well as, protects this traffic against equipment and media failure, must be considered in the design of the modern transmission networks to improve the reliability level.
There are different types of protection architectures [1]:

1. APS (Automatic Protection Switching), usually associated to point-to-point, but can be found in ring and mesh. It can be implemented with different strategies:

- 1:N Protection: "N" working systems share only one protection system. The protection and the working systems can or cannot use the same path;

- 1:1 Protection: for each working system there is a protection one. It can use a Diverse Path (DP) or an Optical Diverse Path (ODP);

- 1+1 Protection: it is similar to the strategy above, but both the systems are working. According to the quality level analysis, one of the two signals is chosen as working.

2. Path Switching: usually associated to unidirectional rings, but can be found in different situations;

3. Line Switching: usually associated to bi-directional rings, using two or four fibers.

According to $\mathrm{Wu}$ [1] and to Beuer \& Hellstöm [2], the $\mathrm{SDH}$ equipment has a modular installation feature that can be viewed in Figure 2.

As a result, the equipment can be divided in optical endings, CPU and signal boards. A TM, for example, can be modeled as an optical ending and a CPU (a "TM rack") and a number of tributaries boards, as well as, an ADM, can be seen as two optical endings and a CPU (an "ADM rack") and a number of add-drop boards. This modularity allows the SDH equipment to be installed in a more efficient way, reducing the system idleness, as well as, permits a more realistic equipment up-grade.

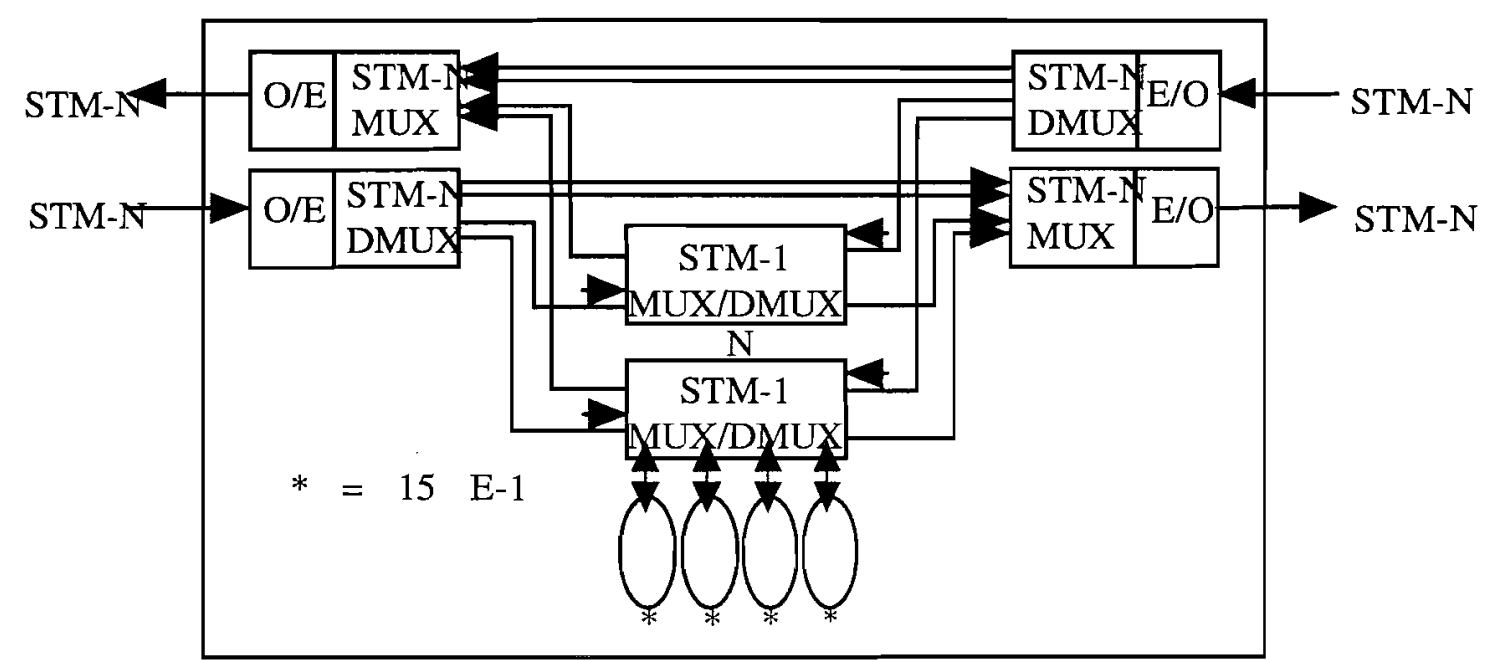

Figure 2 - SDH Equipment 
In this paper, we deal with point-to-point and self-healing ring topologies, using TMs and ADMs. The $34 \mathrm{Mbps}$ (Mega bits per second) PDH systems will also be considere

\section{THE SDH TRANSPORT NETWORK PLANNING}

The telecommunication network planning is a very complex problem, due to the variety of aspects to be considered. As a result, its solving has to be made in many phases:

1. Clustering: the " $n$ " network nodes are split in " $p$ " clusters, trying to join in the same cluster, nodes that have a higher attraction measure. This measure can be the advised sum of traffic interests and/or the inverse of the geographical distance. One node in each cluster is called hub, that usually is the interface between the other nodes of the cluster and the other clusters;

2. Bundling: the necessity of communication between the nodes, i.e., the inter-office trunking, and the trunk routing are used as inputs for finding a collection of topologies that will form the logical network;

3. Physical Network: according to the logical network, obtained in the previous phase, the equipment, as well as, the optical fibers are physically displaced;

\section{Evolution.}

Our interest is the Bundling problem, that can, generally, be seen as a multicommodity network flow optimization problem. The commodities to be routed correspond to the necessity of communication between two Central Offices (COs), that represent the demands of E-1 trunks, i.e., 2 Megabits per second (Mbps) trunks; the arcs of the mathematical model represent the transmission media; the facilities (sets of equipınent forming transmission systems), although physically situated inside the nodes, are associated to the arcs. To describe the network, the arc-chain formulation is adopted.

The solving of the Bundling Problem is made in steps:

1. Facilities assignment: a set of facilities is proposed for constructing the network. This proposal considers the reliability and the survivability aspects through the assignment of different topologies, such as point-topoint, ring, mesh;

2. Mathematical model construction: based on the facilities assigned, a Mixed Integer Linear Programming Model is obtained considering the demand requirements, the equipment data (cost and capacity) and the modular characteristics;

3. Solving the model;

4. Analyzing the results: the step "iii" provides the least cost network that satisfies the problem. However, this solution can not be optimal in an operational way, i.e., this solution can be adapted to become easier and/or more reasonable for implementation, considering network engineering aspects. So, it can be necessary to go to step "ii" and "iii" again;

5. Obtaining the solution for the Bundling Problem.

The major advantages of installing a SDH equipment in a modular way are the systems idleness reduction, the possibility of postponing or anticipating the investments, as well as, the allowance of a more realistic equipment upgrade. For example, a STM-1 ring can be substituted by a STM-4 ring only changing the ADM racks, using the add/drop boards already installed.

The mathematical model for the Static Bundling Problem is:

Minimize

$$
\sum_{j \in L} C_{j} \cdot Q_{j}+\sum_{j \in Z} G_{j} \cdot S_{j}+\sum_{r \in X} C_{r} \cdot Q_{r}+\sum_{p \in H} G_{p} \cdot T_{p}
$$

Subject to

$$
\begin{aligned}
& \sum_{j \in R_{e t}} Y_{j}=d_{e f} \\
& -\sum_{i \in R_{a}} Y_{i}+u_{j} \cdot S_{j}>=0 \\
& -\sum_{j \in L_{a}} m_{j} \cdot Q_{j}+S_{j} \leq 0 \\
& -\sum_{k \in R_{p}} Y_{k}+v_{p} \cdot T_{p}>=0
\end{aligned}
$$

$$
\begin{aligned}
& -\sum_{\mathrm{k} \in \mathrm{R}_{\mathrm{p}}} \mathrm{Y}_{\mathrm{k}}+\sum_{\mathrm{r} \in \mathrm{A}_{\mathrm{i}}} \mathrm{W}_{\mathrm{r}} \cdot \mathrm{Q}_{\mathrm{r}}>=0 \\
& -\sum_{\mathrm{r} \in \mathrm{A}_{\mathrm{i}}} \mathrm{n}_{\mathrm{r}} \cdot \mathrm{Q}_{\mathrm{r}}+\mathrm{T}_{\mathrm{p}} \leq 0
\end{aligned}
$$

$$
-\sum_{l \in B_{v A_{i}}} Y_{I}+\operatorname{CapA}_{i} \geq 0
$$

$$
-\sum_{r \in A_{i}} b_{r} \cdot Q_{r}+\operatorname{CapA}_{i}=0
$$


where, $C_{j}=$ constant associated to the installation cost of the pair " $j$ " of TM racks; $Q_{j}=$ binary variable associated to the installation of the pair " $j$ " of TM racks; $L=$ set of pairs of TM racks to be installed in the network; $G_{j}=$ constant associated to the installation cost of the tributaries board " $\mathrm{j}$ "; $S_{\mathrm{j}}=$ integer variable associated to the number of tributaries boards to be installed in the pair "j" of TM racks; $Z$ = set of tributaries boards to be installed in the network; $C_{T}=$ constant associated to the installation cost of the ADM racks of the ring " $r$ "; $Q_{r}=$ binary variable associated to the installation of the ADM racks of the ring " $r$ "; $X=$ set of $A D M$ racks to be installed in the network; $G_{p}=$ constant associated to the installation cost of the add/drop board " $p$ "; $T_{p}=$ integer variable associated to the number of add/drop boards to be installed in the ADM rack " $\mathrm{p}$ "; $\mathrm{H}=$ set of add/drop boards to be installed in the network; $Y_{j}=$ linear variable associated to the number of E-1 trunks that pass through the chain " $\mathrm{j}$ "; $\mathrm{R}_{\mathrm{ef}}=$ set of chains that attempt the demand of E-1 trunks between the COs "e" and "f"; $d_{e f}=$ constant associated to the demand of E-1 trunks between the COs " $e$ " and " $f$ "; $Y_{i}=$ linear variable associated to the number of E-1 trunks that pass through the chain " $i$ "; $R_{a}=$ set of chains that pass through TMs to be installed in the arc " $a$ "; $\mathrm{u}_{\mathrm{j}}=$ constant associated to the E-1 trunks capacity of each tributaries board " $\mathrm{j}$ "; $\mathrm{m}_{\mathrm{j}}=$ constant associated to the maximum number of tributaries boards that can be installed in TM racks " $\mathrm{j}$ "; $\mathrm{L}_{\mathrm{a}}=$ set of TM racks that can be installed in the arc "a"; $\mathrm{Y}_{\mathrm{k}}=$ linear variable associated to the number of E-1 trunks that pass through the chain " $k$ "; $R_{p}=$ set of chains that pass through bi-directional rings and are added/dropped in the ADM " $p$ "; $v_{p}=$ constant associated to the add/drop capacity E-1 trunks of each add/drop board " $\mathrm{p}$ "; $\mathrm{W}_{\mathrm{r}}=$ constant associated to the add/drop processing capacity of the ADM rack " $r$ "; $A_{i}=$ set of bi-directional rings that can be installed passing through the set " $i$ " of nodes; $n_{r}=$ constant associated to the maximum number of add/drop boards that can be installed in each ADM rack of the ring " $r$ "; $Y_{1}=$ linear variable associated to the number of E-1 trunks that pass through the chain "l"; $\mathrm{B}_{\mathrm{vA}_{\mathrm{i}}}=$ set of chains that pass through the arc " $\mathrm{v}$ " of the set " $\mathrm{i}$ " of rings; $\mathrm{CapA}_{\mathrm{i}}=$ variable associated to the capacity of the set " $i$ " of rings; $b_{r}=$ constant associated to the capacity of the ring "r".

The constraints (1) model the fact that the sum of E-1 =unks that uses all the chains between 2 COs corresponds to the demand between these COs. The constraints (2) and (3) are associated to the point-to-point. The constraints (2) model the fact that the sum of E-1 trunks that uses the chains through some arc has to be smaller than or equal to the capacity of the tributaries boards installed in these TM racks. The constraints (3) model the fact that the tributaries boards can only be installed if the TM rack was installed, and the maximum number of boards has to be smaller than or equal to the rack capacity. The constraints (4), (5), (6), (7) and (8) are associated to the bi-directional self-healing rings. The constraints (4) model the add-drop capacity of each ADM, as a function of the number of add-drop boards installed. The purpose of these constraints is to determine the number of add-drop boards that has to be installed in each ADM rack. The constraints (5) model the add-drop capacity of each $\mathrm{ADM}$, as a function of its processing capacity. The constraints (6) model the fact that the add-drop boards can only be installed if the ADM rack was installed, and the maximum number of boards has to be smaller than or equal to the rack capacity. The constraints (7) model the fact that the sum of E-1 trunks that pass through a ring arc has to be smaller than or equal to the ring transmission capacity. The constraints (8) model the ring transmission capacity.

To consider the equipment modularity aspects, integer variables associated to the signal boards and binary variables associated to the racks are required. Due to this, the mathematical complexity of the model is increased. Trying to reduce it, an optimization tool was developed. It combines a commercial optimization software, the $\mathrm{CPLEX}^{\circledR}$, with a computational procedure, that consists in solving a mixed integer linear problem, with binary, integer and linear variables, through relaxing the integrality constraints, solving the resulting problem, with only binary and linear variables, and applying some heuristics trying to obtain an integer optimal solution. These heuristics are developed to improve the reliability level, considering, preferentially, the variables associated to the add-drop boards. This procedure can be seen in Figure 3.

\begin{tabular}{|c|c|c|c|c|c|c|c|c|c|c|c|c|c|c|c|c|c|c|c|}
\hline A & A & A & A & A & A & B & B & B & B & B & C & C & C & C & D & D & D & E & F \\
- & - & - & - & - & - & - & - & - & - & - & - & - & - & - & - & - & - & - & - \\
B & C & D & E & F & G & C & D & E & F & G & D & E & F & G & E & F & G & F & G \\
\hline 156 & 152 & 144 & 224 & 357 & 29 & 21 & 22 & 18 & 86 & 6 & 19 & 16 & 9 & 20 & 7 & 3 & 5 & 8 & 5 \\
\hline
\end{tabular}

Table 1 - Demands for E-1 trunks 


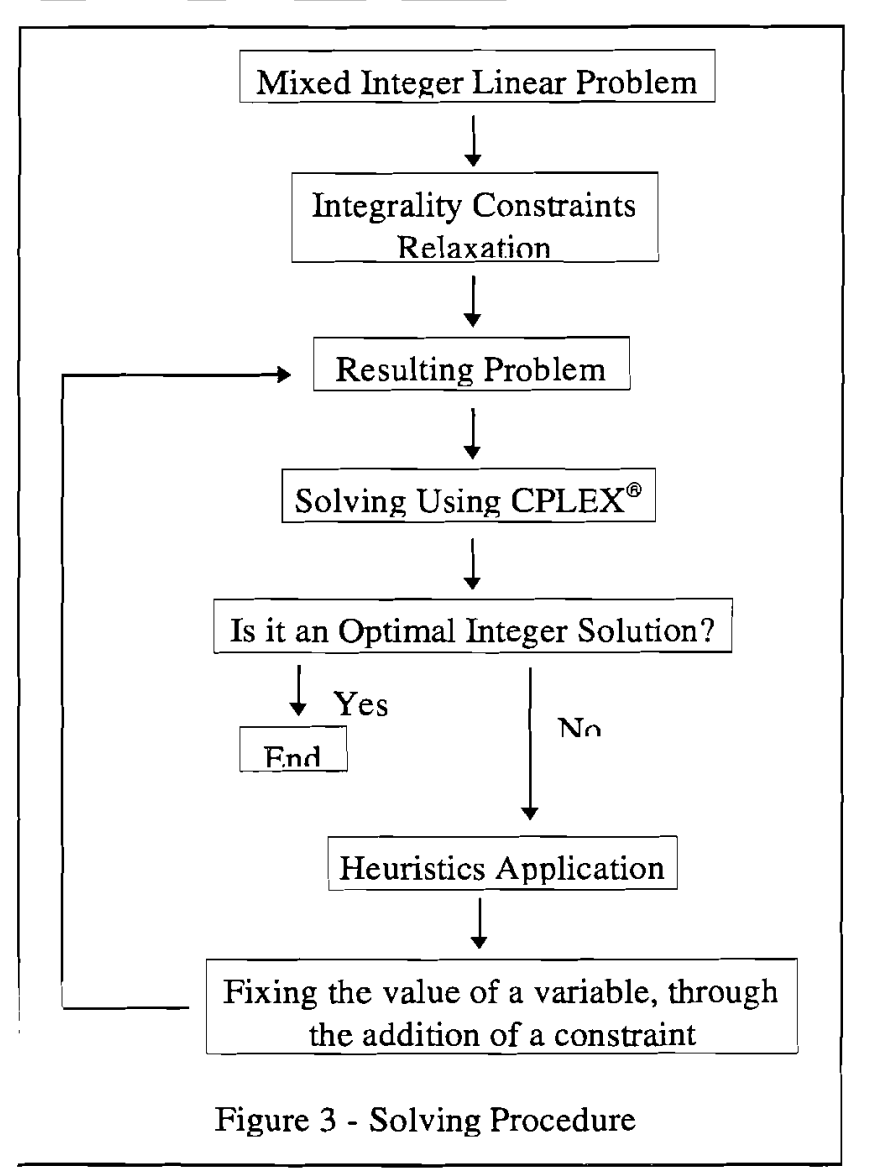

\section{AN APPLICATION EXAMPLE}

As an application example for this methodology, a hypothetical network will be presented. It is based on a Brazilian Medium Size Network. It is defined as:

- the network has 7 nodes (the nodes $\mathrm{A}, \mathrm{B}$ and $\mathrm{C}$ are hubs);

- demands of E-1 trunks, as shown in Table 1.

- the use of TMs and ADMs, respectively, in point-topoint and bi-directional self-healing ring topologies;

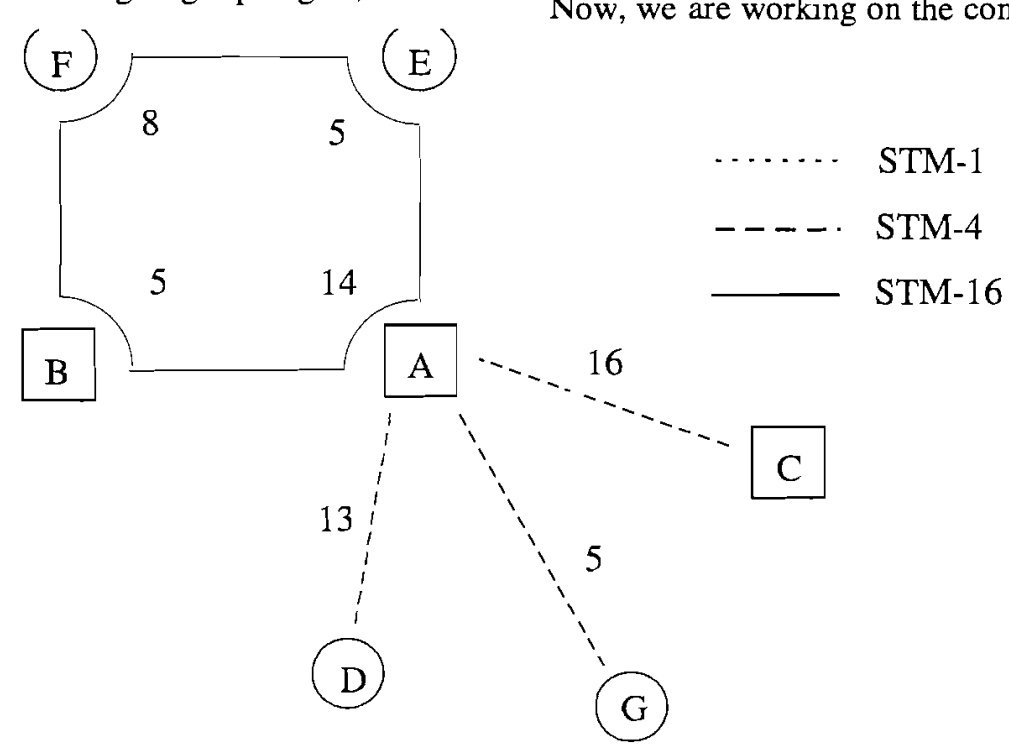

Figure 4 - Solution
- SDH equipment data, as costs (in Monetary Units) and capacities, as shown in Table 2 ;

- the use of $34 \mathrm{Mbps}$ systems (PDH), costing $11 \mathrm{MU}$, with a capacity of $16 \mathrm{E}-1$ trunks.

Applying the developed solving procedure, the network shown in figure 4 was obtained as a solution for the problem, with a cost $=760 \mathrm{MU}$, while the solution obtained with the full equipment installation had a cost $=$ $1120 \mathrm{MU}$. The system idleness, that in some cases used to be more that $40 \%$, decreased to less than $10 \%$. The numbers indicated in the Figure 3 represent:

1. the quantity of add/drop boards installed in each $A D M$ rack in the ring. We can observe that applying the equipment modularity, a different number of add/drop boards can be obtained in a same ring, because this quantity is associated to the required node demand.

2. the quantity of tributaries boards installed in each pair of TM racks.

\section{CONCLUSION}

The modular feature has proved to be very important for the network planning, because the modular equipment installation is more efficient than the full equipment installation. Due to the modularity, the use of integer and binary variables became necessary, that increase the mathematical complexity of the model. The developed solving procedure treats this problem, applying specialized heuristics and solving it, iteratively, as a set of problems with smaller difficulty level, using only binary variables.

We realized that the modular feature can be successfully applied for the Long Range Network Planning, because it is possible to install at a certain stage, only the capacity needed to attempt the demands for this stage, as well as, a more realistic equipment up-grade will be allowed.

Now, we are working on the construction of a Long Rang 
Network Planning Tool, that considers the equipment modular features.

\section{REFERENCES}

[1] T. H. Wu, "Fiber Network Service Survivability", Artech House, 1992.

[2] H. Beuer and B. Hellstöm, "Synchronous Transmission Networks", Ericsson Review, n², pp. 28-39, 1990.

[3] R. D. Doverspike and J. A. Morgan and W. Lelland, "Network Design Sensitivity Studies for Use of Digital Cross-Connect Systems in Survivable Network Architectures", IEEE Journal on Selected Areas in Communications, vol. 12, $\mathrm{n}^{\circ} 1$, pp. 69-78, January 1994.

[4] A. S. Garcia and H. M. F. Tavares, "A Heuristic Method for Digital Trunks Network Planning in Metropolitan Areas: The Use of Optical Fibers", Journal of the Telecommunications Brazilian Society, Vol. 3, n 1, pp. 47-53, December 1988.

[5] I. Baybars and R. H. Edahl, "A Heuristic Method for
Facility Planning in Telecommunications Networks with Multiple Alternate Routes", Naval Research Logistics, Vol. 35, pp. 503-528, 1988.

[6] A. S. Garcia and G. B. Zanandrea, "A Heuristic Approach for SDH Transmission Network Evolution", proceedings of the 4th International Conference on Telecommunications Systems, pp. 620-626, 1996.

[7] S. Bortolon and H. M. F. Tavares and R. V. Ribeiro, "SONET Planning Using Optimization Tools in Large Networks: A Practical Case Study", proceedings of the 4th International Conference on Telecommunications Systems, pp. 493-499, 1996.

[8] A. S. Garcia and G. B. Zanandrea, "The Use of Heuristics Methods for Optimized Evolution of SDH Transmission Networks", proceedings of the 11th Brazilian Conference on Automatics, pp. 239-244, 1996.

[9] Using the CPLEX ${ }^{\oplus}$ Callable Library, CPLEX Optimization, Inc., 1995.

Table 2 - SDH Equipment Data

\begin{tabular}{|c|c|c|c|}
\hline & $\begin{array}{c}155 \mathrm{Mbps} \\
\text { (STM-1) }\end{array}$ & $\begin{array}{l}622 \mathrm{Mbps} \\
\text { (STM-4) }\end{array}$ & $\begin{array}{l}2.48 \mathrm{Gbps} \\
\text { (STM-16) }\end{array}$ \\
\hline Cost of each TM rack & 22 & 28 & 32 \\
\hline Cost of each ADM rack & 42 & 58 & 74 \\
\hline Transmission capacity (E-1 trunks) & 63 & 252 & 1008 \\
\hline Add-drop processing capacity (E-1 trunks) & 126 & 504 & 2016 \\
\hline Maximum number of tributaries boards in each TM rack & 4 & 16 & 64 \\
\hline Maximum number of add-drop boards in each ADM rack & 2 & 8 & 32 \\
\hline \multicolumn{4}{|l|}{ Cost of each tributaries board $=1$} \\
\hline \multicolumn{4}{|l|}{ Cost of each add-drop board $=4$} \\
\hline \multicolumn{4}{|l|}{ Capacity of each tributaries board $=15 \mathrm{E}-1$ trunks } \\
\hline Capacity of each add-drop board $=63 \mathrm{E}-1$ trunks & & & \\
\hline
\end{tabular}


Anilton Salles Garcia was born in Afonso Claudio, ES, Brazil, on September 14, 1953. He received the degree of Mechanical Engineer from Federal University of Espirito Santo in 1976, the M. Sc. degree in Applied Mathematics: Optimization and Operations Research from IMECC - State University of Campinas in 1978 and the D. Sc. degree in Electrical Engineering: Automation from FEE - State University of Campinas in 1987. Since 1978, he is an Associate Professor at Federal University of Espirito Santo. From 1987 to 1990 , he was a Planning Engineer at Elebra Telecom Ltda. He was a research assistant in a Cooperation Program between State University of Campinas and Telebras Research and Development Center (CPqD - Telebras) from 1981 to 1986. His research interests are telephone traffic studies, telecommunications systems design, SDH networks, digital network planning, ATM switching networks and Intelligent networks.

Gustavo Balestrero Zanandrea was born in Vitoria, ES, Brazil, on November 23, 1972. He received the degree of Electrical Engineer from Federal University of Espirito Santo in 1994. In 1996, he joined the engineering staff of Vale do Rio Doce Company (CVRD). $\mathrm{He}$ is currently working toward his $\mathbf{M}$. Sc. degree in Electrical Engineer: Automation. His research interests are telecommunication system design, SDH networks, digital network planning and ATM switching networks. 